\title{
Maximization of performance of a PCM - based thermal energy storage systems
}

\author{
Marta Kuta $^{1 *}$, Dominika Matuszewska ${ }^{1}$ and Tadeusz M. Wójcik ${ }^{1}$ \\ ${ }^{1}$ AGH University of Science and Technology, Faculty of Energy and Fuels, Department of Thermal and Fluid Flow Machines, \\ al. A. Mickiewicza 30, 30-059 Krakow
}

\begin{abstract}
Phase change materials (PCMs) are significant in terms of applicability for the thermal energy storage (TES). Thanks to the high thermal storage density and wide range of phase transition temperature they are promising storage mediums for a large number of applications. PCMs can be used to support efficient use of waste or excess heat. Selection of adequate material as well as design of optimal TES magazine are crucial. It is important to choose material which is characterized by suitable temperature range of phase transition, possibly high latent heat of transition, specific heat and thermal conductivity. Also important features are: ability to work properly after many operation cycles, minimum volume change and gas generation during the phase transition. It is also advantageous when PCM is non-toxic and noncorrosive, non-flammable, non-explosive, environment friendly and easy to recycle. Even the best designed PCMs would not be able to store heat efficiently if the whole magazine and its construction were not good enough. This is the reason why a lot of effort is taken to design effective TES system. The aim of this work is to analyse examples of different configurations of PCM - based thermal energy storage systems. Authors compare selected TES systems and discuss their characteristics.
\end{abstract}

\section{Introduction}

Thermal energy storage is an important issue in number of areas, including: renewable energy, domestic and industrial uses of heat or thermal stabilisation of chemical and industrial processes. Storing of waste or excess heat allows its later use. It helps to reduce or remove the gap between heat generation and consumption and at the same allows cost savings. In addition - TES can be used as a support for temperature control which is critical to many processes in order to ensure their efficiency.

Thermal energy can be stored by one of the listed methods: sensible heat storage, latent heat storage, thermochemical storage.

Sensible heat storage consists of heat accumulation as a result of the medium temperature increase. Main factors determining amount of accumulated heat are: amount of the storage medium, specific heat and the temperature change.

Latent heat storage consists of two parts: first one involve heating or cooling material to the specific temperature of phase transition, which is typical for particular PCM and second - main part - uses change of phase of storage medium. In this case factors determining amount of accumulated heat are: specific latent heat, amount of material and fraction of transformed material.

The last method of TES is thermochemical heat storage, which is based on reversible endothermic chemical reactions. First two methods and their combination are more commonly used due to its higher availability and development.

The objective of this work is to review the recent technologies of thermal energy storage for various applications, with the use of phase change materials, whose work is based on the use of latent heat.

Phase change material can use one of listed types of transition: solid - liquid, liquid - solid or solid - solid. The widest selection represent materials from the group of solid - liquid PCMs. This kind of transformation has been well studied and materials are easily accessible and operated for many applications. Some applications require that, there is no liquid phase in any stages of transition. In that case it is necessary to contain or encapsulate PCMs in order to avoid the leakage into the system. Alternative solution is to use solid-solid PCMs, which are characterized by a lack of liquid or gaseous phase and the lack of volume change during the transition. However they are also characterized by smaller storage capability. The highest latent heat of phase change is typical for liquid - gas PCMs. Despite that, this it is not the most popular group of phase change materials. They are not practical and uneasy to use because of its enormous change of volume and the presence of gas phase.

Phase change material seem to have a great potential and wide range of possible application. It is important to take into consideration that any application need carefully selected PCMs which include all requirements.

Corresponding author: marta.kuta@agh.edu.pl 
It is also important to remember about every disadvantage of phase change materials, among which the most important is low thermal conductivity. It cause limitations on its use. In order to reduce mentioned problems, many techniques have been developed, also in thermal energy storage. They will be described more precisely in the following sections.

\section{Phase change materials for thermal energy storage}

Thermal energy storage with the use of PCMs is more effective than sensible heat storage. Phase change materials can store 5-14 times more heat (per volume unit) than materials which work is based on sensible heat [1]. There is a condition that have to be complied for the effective heat storage: Phase change material need to be characterized by specific properties and properly selected for the application. Number of properties should be considered, most important are [2]:

- suitable temperature of phase transition,

- high latent heat of transition,

- high specific heat,

- high thermal conductivity,

- high density,

- small or no volume change,

- minimal gas generation during the transition,

- no degradation and stability of properties after many cycles,

- minimal subcooling,

- non-corrosive,

- compatibility with the system/construction,

- non-flammable,

- non-explosive,

- easy to recycle,

- environment friendly,

- availability,

- cost-effective.

Usually it is not possible to meet all listed requirements. However, it is important to choose the material closest to the needs of application. It is necessary that PCM use was reasonable to use material with suitable for application temperature of phase transition. High latent heat of transition allows to use smaller amount of material, additionally high specific heat influence higher sensible heat storage. High thermal conductivity increase phase transition process. High density, as well as small volume change and small gas generation and compatibility with the system enable easier use and selection of container or heat exchanger construction. For safety it is also important that PCMs are noncorrosive, non-flammable, non-explosive and environment friendly. From the economical point of view it is important to select not expensive and easly available material.

Table 1 consists selected manufacturers of phase change materials with information about the range of transition temperatures of PCMs available in those companies. As it can be seen, wide range of PCMs are commercially available.
Table 1. Selected companies manufacturing PCMs.

\begin{tabular}{|c|c|c|}
\hline Manufacturer & Melting point, ${ }^{\circ} \mathrm{C}$ & Ref. \\
\hline BASF & $21,23,26$ & [3] \\
\hline Climator Sweden AB & $-21-70$ & [4] \\
\hline $\begin{array}{l}\text { CRISTOPIA Energy } \\
\text { Systems }\end{array}$ & $-33-27$ & [5] \\
\hline Croda International Plc & $5-53$ & [6] \\
\hline Cryopak Inc & $5-22$ & [7] \\
\hline Laird PLC & $-25-125$ & {$[8]$} \\
\hline PCM Energy P. Ltd & $-50-89$ & [9] \\
\hline $\begin{array}{l}\text { Phase Change Material } \\
\text { Products Limited }\end{array}$ & $-100-885$ & {$[10]$} \\
\hline $\begin{array}{l}\text { Pluss Advanced } \\
\text { Technologies Pvt. Ltd. }\end{array}$ & $-33-89$ & [11] \\
\hline Rgees LIC & $-26-65$ & [12] \\
\hline $\begin{array}{l}\text { Rubitherm Technologies } \\
\text { GmbH }\end{array}$ & $-21-90$ & [13] \\
\hline Salca BV & $5-90$ & [14] \\
\hline
\end{tabular}

There are also numbers of scientists conducting their research in the area of phase change materials for thermal energy storage. Rathod and Banerjee [15] summarised in their work number of tests performed on Phase change materials. They compare, based on the literature, thermal stability of PCMs from different groups: organic (paraffins and non-paraffins), inorganic (salt hydrates and metallic) and eutectics. Few points based on their summary have been chosen and listed below:

- Thermal and chemical stability as well as corrosion resistance influence the length of PCMs life

- The biggest number of investigated organic and inorganic PCMs are characterised by phase change transition temperature in the range of $30-60{ }^{\circ} \mathrm{C}$. For eutectics it looks similar: $20-60^{\circ} \mathrm{C}$.

- Paraffins as representant of organic PCMs usually are stable after many work cycles. Salt hydrates from the inorganic group of PCMs have problems with phase separation and supercooling, but both disadvantages can be reduced with the use of well-known methods.

- Method which is the most commonly used by researchers for determination of PCMs fundamental properties is differential scanning calorimetry.

One of the biggest problem with the use of phase change materials, both: commercial and non-commercial is low 
thermal conductivity. Number of researchers work on new methods on enhancing thermal conductivity of PCMs for thermal energy storage. Khodadadi et al. [16] collect in their work a review of researches in this area. They focused on nanotechnology in PCMs thermal energy storage. Selected conclusions are listed below:

- Numbers of nanostructures are used to increase thermal conductivity of PCMs, including: carbon-based nanostructures, carbon nanotubes, metallic and metal oxide, nanoparticles and silver nanowires

- Results for addition of carbon based nanostructures or carbon nanotubes are prominent in comparison with metallic nanoparticles.

\section{PCM Containers and TES tank}

The geometry and thermal parameters of PCM container for thermal energy storage are crucial. As it has been already described PCMs have very low thermal conductivity. This section will discuss another solution for increasing thermal conductivity of PCMs system than addition of nanoparticles. Discussion will be focused on aspects related to PCM containers and TES tanks.

The most commonly used geometrical configurations of TES tank are: cylinder (tube in shell, packet bed) and rectangle. Several study have been also carried out to determine the most effective constructions of PCM container. Some of research in both areas and commercial products are described below.

Table 2 presents examples of containers and capsules commercially available and suitable for thermal energy storage applications. Variety of containers gives possibility to select the one which meets most expectations regarding to: size, construction, material, operating temperature, heat transfer rate or economical aspects.

Table 2 Examples of commercially available containers and macrocapsules filled with PCMs

\begin{tabular}{|c|c|c|}
\hline PCM container/ & Manufacturer & Ref. \\
\hline $\begin{array}{c}\text { Eap } \\
\text { Eutectic liquid (PCM) }\end{array}$ & $\begin{array}{c}\text { CRISTOPIA } \\
\text { Energy } \\
\text { Systems }\end{array}$ & {$[5]$} \\
Enveloppe in polyolefines & & \\
Nodule filled with PCM & & \\
\hline PCM Rubber Balls & $\begin{array}{c}\text { Phase Change } \\
\text { Material } \\
\text { Products }\end{array}$ & {$[10]$} \\
\hline Limited & \\
\hline PCM & & \\
\hline
\end{tabular}

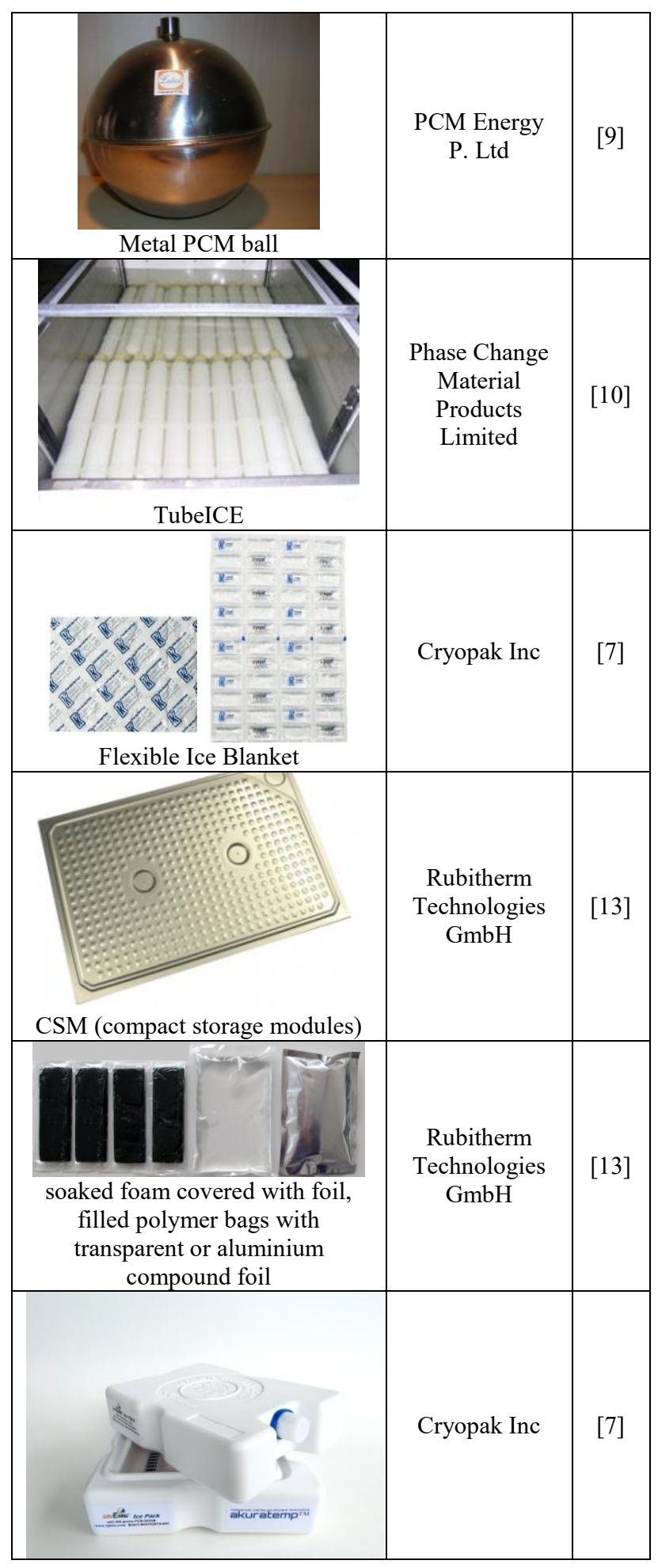

Castell et al. [17] present in their work experimental study of a PCM tank for cold storage. They analyse two different configurations and two different flow rates of the heat transfer fluid. Figure 1 and 2 show two experimental configuration: figure 1 - with higher packing factor, figure 2 - with higher heat transfer surface. 

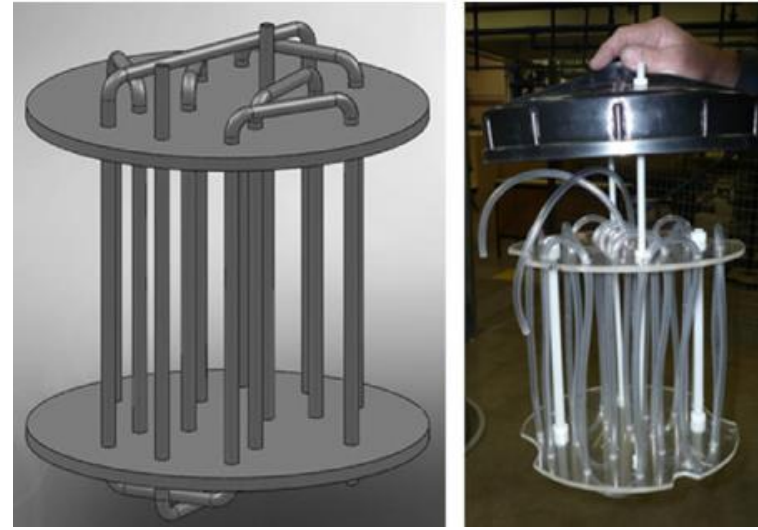

Figure 1 Schematic and photo of the experimental PCM tank with higher packing factor[17]
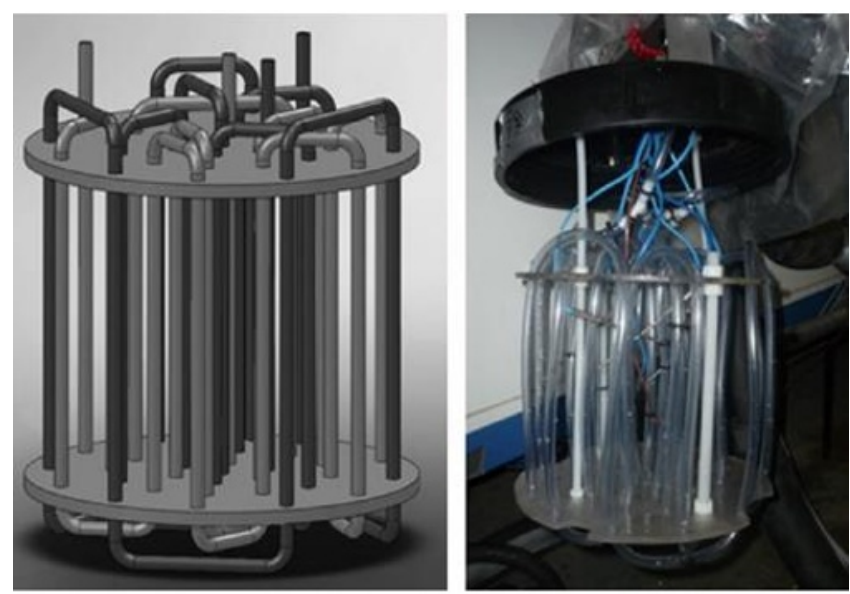

Figure 2 Schematic and photo of the experimental PCM tank with high heat transfer surface [17]

Authors concluded that: effectiveness of heat exchange of the system did not vary with time, it decrease with increasing flow rate and increase with increasing heat transfer area.

López-Navarro et al. [18] presents in their work experimental results of a versatile latent heat storage tank capable of working with organic phase-change materials within a temperature range from $10^{\circ} \mathrm{C}$ to $100^{\circ} \mathrm{C}$.

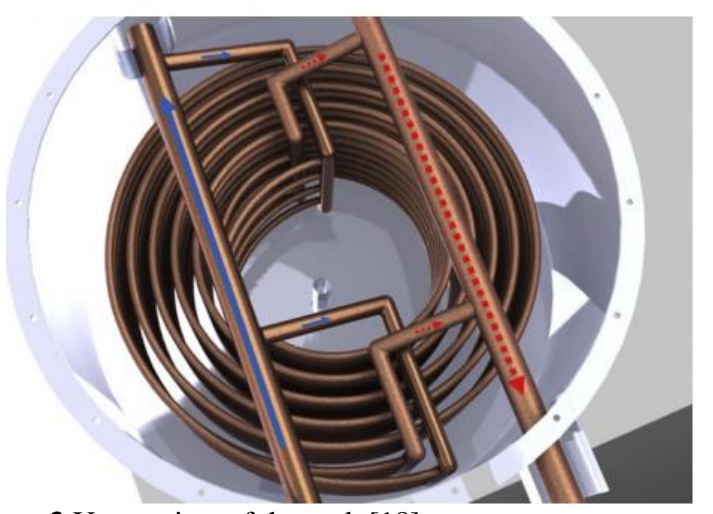

Figure 3 Upper view of the tank [18]

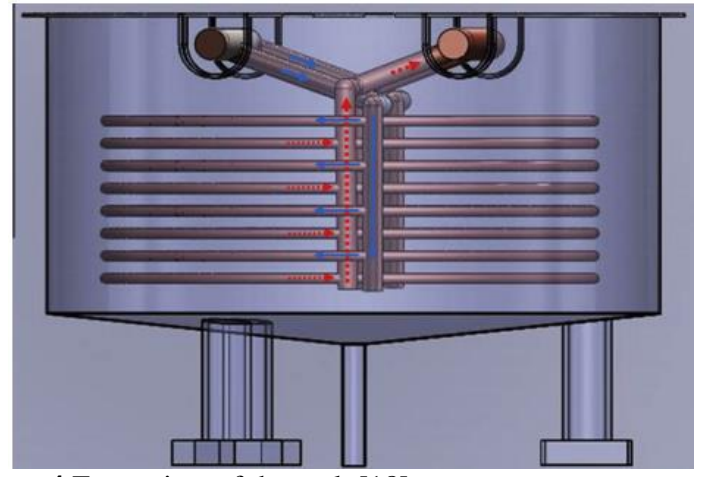

Figure 4 Front view of the tank [18]

Authors calculated the average effectiveness, the phase change fraction and the total heat transfer. The results have shown that up to $78 \%$ of the maximum capacity can be reached within $4 \mathrm{~h}$, and that the stored energy is more sensitive to supply temperature variations in the melting tests.

Aldos and Rahman et al. [19] compare in their work single-PCM and multi-PCM thermal energy storage design. They use spherical capsules filled with PCMs on different thermo-physical properties. The capsules are packed in the bed at different sections based on the PCM melting temperature which match the heat transfer fluid (HTF) temperature. Authors calculated and presented the performance of the system in terms of charging and discharging rate, rate of heat transfer and storage capacity.

Figure 5 shows the schematic system - packed bed with PCM capsules.

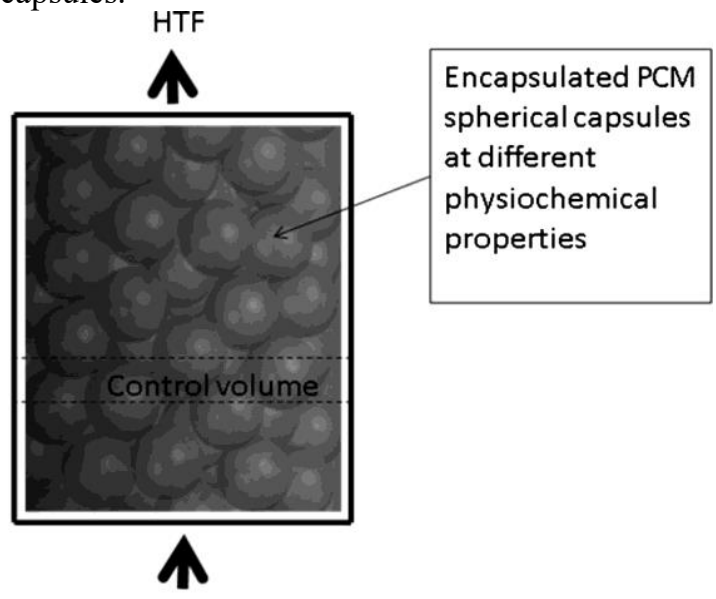

Figure 5 Schematics of the LTES system [19]

Authors concluded that multi-PCM thermal energy storage system perform better than single-PCM design. They also took noticed that performance for more than three stages does not results with meaningful additional results.

\section{Mathematical and numerical modelling}

Selection of the PCM container/TES tank, proper construction is difficult and complex work. Several 
researchers decided to use mathematical and numerical modelling to simplify and shorten the experiments. Some examples are discussed below.

Sciacovelli et al. [20] combined in their work CFD modelling with the response surface method. The aim of their work was to optimize the shape of fins to maximize the performance of the system. Figure 6 shows the system.

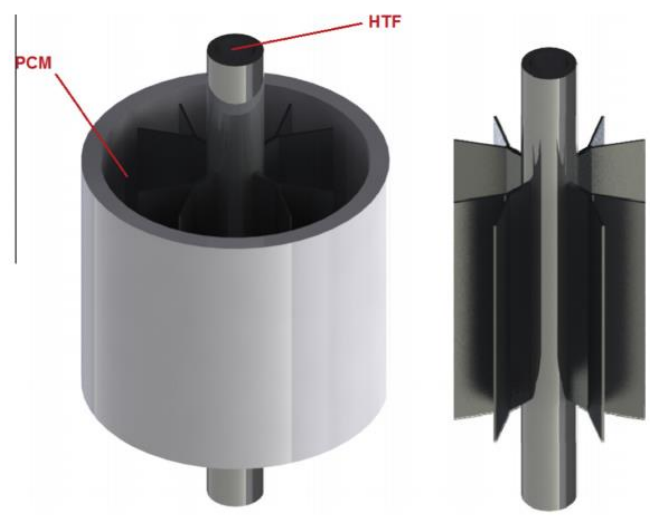

Figure 6 System analysed by Sciacovelli et al. [20], (HTF heat transfer fluid)

Figure 7 shows the fin configuration.

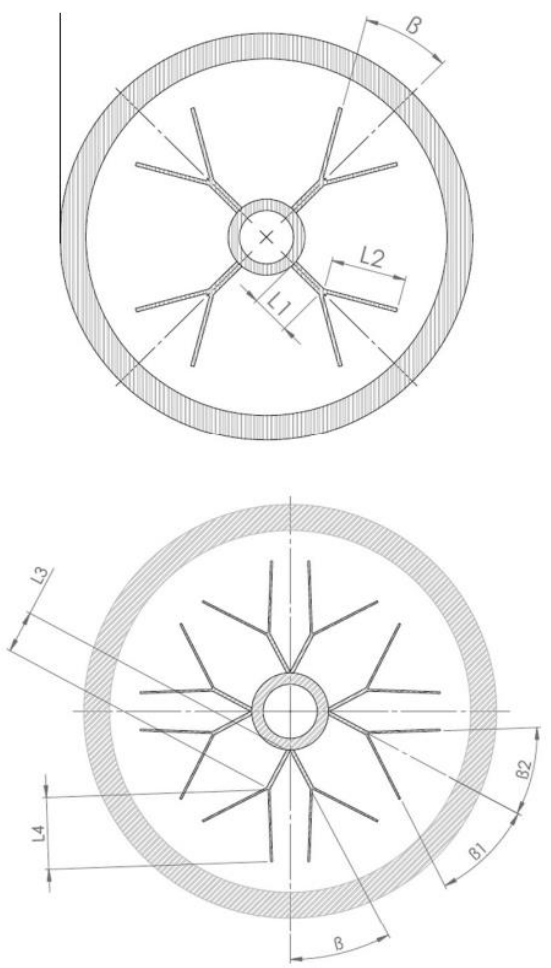

Figure 7 Fin configuration: single bifurcation (top), double bifurcation (bottom) [20]

Authors conclude that discharge efficiency results for optimal tree shaped fins increase of about $24 \%$. They also noticed that different fin design is optimal according to the system operating time. For short operating time fins with wide angles between branches are more effective, but for long operating time smaller angles are necessary.

Chow et al. [21] investigated by two thermal conductivity enhancement techniques:

- use different types of containers to encapsulate PCM and place them in a liquid metal medium, - use a metal/PCM composite.

Figure 8 presents six type of investigated containers and the dimension of all configuration is listed in table 3
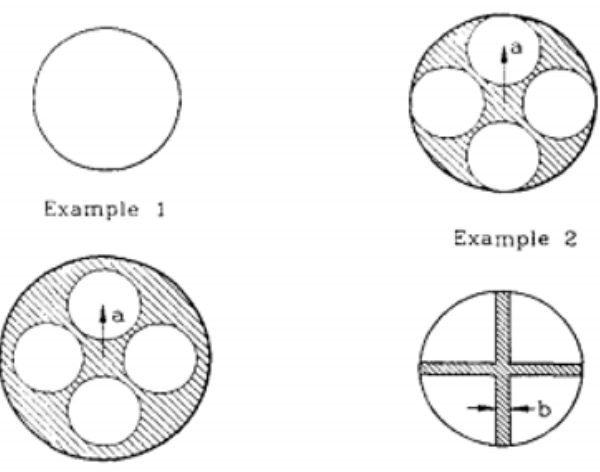

Example 2

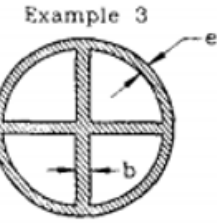

Example 5

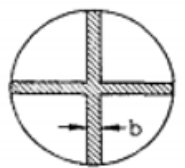

Exampie 4

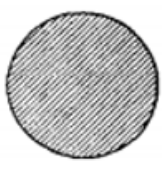

Example 6
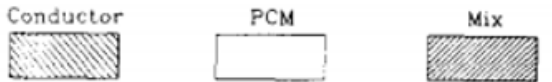

Figure 8 Thermal energy storage configuration[21]

Table 3 Dimensions of containers [21]

\begin{tabular}{|l|l|l|l|l|}
\hline Example & $\mathrm{d}, \mathrm{cm}$ & $\mathrm{a}, \mathrm{cm}$ & $\mathrm{b}, \mathrm{cm}$ & $\mathrm{e}, \mathrm{cm}$ \\
\hline 1 & 4 & & & \\
\hline 2 & 5 & 1,5 & & \\
\hline 3 & 5,75 & 1,75 & & \\
\hline 4 & 4,5 & & 0,37 & \\
\hline 5 & 5 & & 0,37 & 0,25 \\
\hline 6 & 4 & & & \\
\hline
\end{tabular}

Results confirmed that both analysed methods can influence the thermal conductivity of PCMs. Using composites gives better effect.

\section{Summary}

The use of phase-change materials for thermal energy storage offers many opportunities to increase the amount of stored heat. However, it is important to pay attention to challenges associated with their use. One of them is low thermal conductivity. Many tests are carried out to 
improve this PCM's property. An additional way to improve the use of phase-change materials for thermal energy storage is using a suitable for application heat exchanger design. Selected examples of possible constructions have been identified and discussed in this paper. Individual constructions should be adjusted depending on the selected PCM and the operating mode of the system. Each of the indicated structures is characterized by the advantages and disadvantages listed in the article. This area requires further detailed research.

\section{Acknowledgement}

This work was supported by AGH - University of Science and Technology (Project 11.11.210.376).

\section{References}

1. A. Sharma, V.V Tyagi, C.R. Chen, D. Buddhi, Renewable and Sustainable Energy Reviews 13, (2009) 318-345

2. M. Kuta, D. Matuszewska, T. M. Wójcik Web of Conferences 10 (2016) 00068

3. BASF at www.micronal.de available on $10^{\text {th }}$ September 2016

4. Climator Sweden $\mathrm{AB}$ at www. climator.com available on $10^{\text {th }}$ September 2016

5. CRISTOPIA Energy Systems www.cristopia.com available on $10^{\text {th }}$ September 2016

6. Croda International Plc at www.crodaindustrialchemicals.com available on $10^{\text {th }}$ September 2016

7. Cryopak Inc at www.cryopak.com available on $10^{\text {th }}$ September 2016

8. Laird PLC at www.lairdtech.com available on $10^{\text {th }}$ September 2016

9. PCM Energy P. Ltd at www.pcmenergy.com available on $10^{\text {th }}$ September 2016

10. Phase Change Material Products Limited at www.pcmproducts.net available on $10^{\text {th }}$ September 2016

11. Pluss Advanced Technologies Pvt. Ltd. at www.pluss.co.in available on $10^{\text {th }}$ September 2016

12. Rgees LIC at www.rgees.com available on $10^{\text {th }}$ September 2016

13. Rubitherm Technologies $\mathrm{GmbH}$ at www.rubitherm.eu available on $10^{\text {th }}$ September 2016

14. Salca BV at www.salcabv.nl available on $10^{\text {th }}$ September 2016

15. M.K. Rathod, J. Banerjee, Renewable and Sustainable Energy Reviews 18 (2013) 246-258

16. J.M. Khodadadi, L. Fan, H. Babaei, Renewable and Sustainable Energy Reviews 24 (2013) 418-444

17. A. Castell, M. Belusko, F. Bruno, L.F. Cabeza, Applied Energy 88 (2011) 4120-4127

18. A.López-Navarro, J. Biosca-Taronger, J.M. Corberán, C. Peñalosa, A. Lázaro, P. Dolado, J. Payá, Applied Energy 119 (2014) 151-162
19. T. K. Aldoss, M.M. Rahman, Energy Conversion and Management 83 (2014) 79-87

20. A. Sciacovelli, F. Gagliardi, V. Verda, Applied Energy 137 (2015) 707-715

21. L.C. Chow and J.K. Zhong, International Communications in Heat and Mass Transfer, 23 (1996) 91-100 